LYCEN 2002-31

June 14th, 2002

\title{
Improved Exclusion Limits from the EDELWEISS WIMP Search
}

\author{
The EDELWEISS Collaboration:
}

\author{
A. Benoit ${ }^{1}$, L. Bergé2 ${ }^{2}$, A. Broniatowski², L. Chabert ${ }^{3}$, B. Chambon ${ }^{3}$, \\ M. Chapellier ${ }^{4}$, G. Chardin ${ }^{5}$, P. Charvin ${ }^{5,6}$, M. De Jésus ${ }^{3}$, P. Di Stefano ${ }^{3}$, \\ D. Drain ${ }^{3}$, L. Dumoulin ${ }^{2}$, J. Gascon ${ }^{3}$, G. Gerbier ${ }^{5}$, E. Gerlic ${ }^{3}$, \\ C. Goldbach 7 , M. Goyot 3 , M. Gros 5 , J.P. Hadjout ${ }^{3}$, S. Hervé ${ }^{5}$, A. Juillard ${ }^{2}$, \\ A. de Lesquen ${ }^{5}$, M. Loidl ${ }^{5}$, J. Mallet ${ }^{5}$, S. Marnieros ${ }^{2}$, O. Martineau ${ }^{3}$,

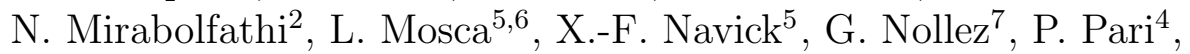 \\ C. Riccio ${ }^{5,6}$, V. Sanglard ${ }^{3}$, M. Stern ${ }^{3}$, L. Vagneron ${ }^{3}$ \\ ${ }^{1}$ Centre de Recherche sur les Très Basses Températures, SPM-CNRS, BP 166, 38042 Grenoble, France \\ ${ }^{2}$ Centre de Spectroscopie Nucléaire et de Spectroscopie de Masse, IN2P3-CNRS, Université Paris XI, bat \\ 108, 91405 Orsay, France \\ ${ }^{3}$ Institut de Physique Nucléaire de Lyon-UCBL, IN2P3-CNRS, 4 rue Enrico Fermi, 69622 Villeurbanne \\ Cedex, France \\ ${ }^{4}$ CEA, Centre d'Études Nucléaires de Saclay, DSM/DRECAM, 91191 Gif-sur-Yvette Cedex, France \\ ${ }^{5}$ CEA, Centre d'Études Nucléaires de Saclay, DSM/DAPNIA, 91191 Gif-sur-Yvette Cedex, France \\ ${ }^{6}$ Laboratoire Souterrain de Modane, CEA-CNRS, 90 rue Polset, 73500 Modane, France \\ ${ }^{7}$ Institut d'Astrophysique de Paris, INSU-CNRS, 98 bis Bd Arago, 75014 Paris, France
}

\section{Abstract}

The EDELWEISS experiment has improved its sensitivity for the direct search for WIMP dark matter. In the recoil energy range relevant for WIMP masses below $10 \mathrm{TeV} / \mathrm{c}^{2}$, no nuclear recoils were observed in the fiducial volume of a heat-and-ionization cryogenic Ge detector operated in the lowbackground environment of the Laboratoire Souterrain de Modane in the Fréjus Tunnel, during an effective exposure of $7.4 \mathrm{~kg} \cdot \mathrm{d}$. This result is combined with the previous EDELWEISS data to derive a limit on the crosssection for spin-independent interaction of WIMPs and nucleons as a function of WIMP mass, using standard nuclear physics and astrophysical assumptions. This limit excludes at more than 99.8\%CL a WIMP candidate with a mass of $44 \mathrm{GeV} / \mathrm{c}^{2}$ and a cross-section of $5.4 \times 10^{-6} \mathrm{pb}$, as reported by the DAMA collaboration. A first sample of supersymmetric models are also excluded at $90 \%$ CL. 


\section{Introduction}

The experimental efforts in the search for Cold Dark Matter in the form of Weakly Interacting Massive Particles (WIMPs) are steadily increasing (see e.g. Ref. [1] for a review). In direct searches, the experimental signature of the WIMPs from the galactic halo is the observation of nuclear recoils induced by their scattering. Current experimental sensitivities for the interaction rate of WIMPs are of the order of 1 per kilogram detector material and per day for the various experiments at the forefront of this search [8]-[8].

The experiment DAMA [3] has reported an annual modulation signal in NaI detectors. This represents a challenge to other detecting methods to reach equivalent sensitivities, a standard procedure to compare different experiments having been laid out in Ref. [9]. Two experiments[圆, 8], both using cryogenic heat-and-ionization germanium detectors, were able to exclude at more than 90\%CL the central value deduced by DAMA from its annual modulation signal for the WIMP mass and its nucleon scattering cross-section $\left(M_{W}=52 \mathrm{GeV} / \mathrm{c}^{2}\right.$ and $\sigma_{n}=7.2 \times 10^{-6} \mathrm{pb}$, respectively). The CDMS experiment [4] was the first to report a limit excluding this value. However, the operation of the detectors in a shallow site, with only 16 meters of water equivalent (m.w.e.) protection from cosmic rays, leads to a sizable background of nuclear recoils from neutron scattering that requires a delicate procedure of background subtraction [0]. The EDELWEISS experiment [8], located in a 4800 m.w.e. deep underground site, was also able to reject that value without requiring any background subtraction. However, the energy resolution in that experiment restricted the sensitivity to nuclear recoils above $30 \mathrm{keV}$ and the accumulated run time was not sufficient to extend the sensitivity to the central value obtained by DAMA [3] when their annual modulation signal is combined with their exclusion limit from pulse shape discrimination in $\mathrm{NaI}$ [2] $\left(M_{W}=44 \mathrm{GeV} / \mathrm{c}^{2}\right.$ and $\left.\sigma_{n}=5.4 \times 10^{-6} \mathrm{pb}\right)$. Beyond this, an important step in these searches would be to reach out to the Supersymmetric model calculations predicting the largest $\sigma_{n}$ values.

Following the results obtained by EDELWEISS using a heat-and-ionization cryogenic Ge detector [8], three new detectors were put in operation. The aim was to improve our understanding of the performance of such detectors, and to extend the sensitivity to lower cross-sections. This letter presents the improved cross-section limit achieved using a detector with improved charge collection and energy resolution.

\section{Experimental Setup}

The experiment is located in the Laboratoire Souterrain de Modane in 
the Fréjus Tunnel under the French-Italian Alps, under a 4800 m.w.e. rock overburden. The experimental setup is described in [8]; only the relevant modifications are discussed here.

Three new 320 g cryogenic Ge detectors [10], each $70 \mathrm{~mm}$ in diameter and $20 \mathrm{~mm}$ in height, are operated simultaneously. Each one is equipped with a segmented electrode defining two regions, a central part and a guard ring. To improve their reliability, all electrical contacts with the electrodes are ultrasound-bonded instead of glued as in Ref. [8].

Two of the detectors, labeled GeAl9 and GeAl10, are very similar to the one used in the year 2000 runs (Ref. [8]), labeled GeAl6. The third detector, GGA1, differs by the presence of a $60 \mathrm{~nm}$ hydrogenated amorphous Ge layer deposited under the $70 \mathrm{~nm} \mathrm{Al}$ electrodes and on all exposed surfaces. This modification was done to test whether an amorphous layer can improve charge collection properties, as suggested by [12]. Bias voltage values between 2 and $4 \mathrm{~V}$ are used.

The size of the NTD heat sensors and the thermalization of the detectors are improved for a better sensitivity, in light of the previous experience with GeAl6. As a result, it was possible to operate the detector at a reduced temperature of $17 \mathrm{mK}$ (regulated to within $10 \mu \mathrm{K}$ ).

The data acquisition system has been upgraded to a design with fully numerical data flow and trigger. The signals from the 3 heat and 6 ionization channels are continuously digitized at respective rates of 2 and $200 \mathrm{ksample} / \mathrm{s}$ and sent to the data acquisition $\mathrm{PC}$ via an optical link. The ionization data are then filtered on-line using an Infinite Impulse Response (IIR) high-pass elliptic filter of 4th order, in order to remove most of the microphonics noise, below a frequency of $1200 \mathrm{~Hz}$. The trigger is defined by requiring a minimum threshold on the absolute value of any of the filtered ionization channels.

\section{Detector Calibration}

The heat and ionization responses to $\gamma$ rays were calibrated using ${ }^{57} \mathrm{Co}$ and ${ }^{60} \mathrm{Co}$ sources. In 2000 [8], the performance of the detector GeAl6 was partly limited by a poor baseline resolution on both ionization and heat channels. While resolutions at $122 \mathrm{keV}$ of the new detectors remained close to those of GeAl6, the baseline resolutions were somewhat improved. The ionization baseline resolutions are all below $1.5 \mathrm{keV}$ FWHM, and are 1.3, 0.5 and $0.4 \mathrm{keV}$ for the heat channels in GGA1, GeAl9 and GeAl10, respectively. The resulting improvement is illustrated in Fig. 1, showing the low-energy spectra recorded in the three detectors in the low-background physics run. Here, the energy corresponds to the average of the ionization and heat signals, weighted by the square of their respective resolutions. The 8.98 and 10.37 
$\mathrm{keV}$ lines from the decay of the cosmic-ray induced long-lived isotopes ${ }^{65} \mathrm{Zn}$ and ${ }^{68} \mathrm{Ge}[13$ are clearly resolved in GeAl9 and GeAl10, with a resolution of $0.6 \mathrm{keV}$ FWHM. The resolution in GGA1 is only $1.2 \mathrm{keV}$ FWHM, but the two-peaked structure can again be observed. The degraded resolution of GGA1 relative to the two other detectors is due to a reduced NTD sensor volume (1.6 vs $5.6 \mathrm{~mm}^{3}$ ), and an increased sensitivity to microphonics of the center electrode ionization channel.

The threshold level of the ionization trigger was measured using two different techniques. The first one consists in extracting, as in Ref. [8], the threshold value corresponding to an efficiency of $50 \%$ from a fit to the lowenergy part of the Compton plateau recorded with a ${ }^{60} \mathrm{Co} \gamma$-ray source. The second technique was made possible by the simultaneous operation of the three detectors with a ${ }^{252} \mathrm{Cf}$ neutron source. Neutron scattering induces a large number of coincidence events where at least two detectors are hit. The efficiency curve as a function of ionization energy in one detector is given by the ratio of the ionisation energy distributions obtained with and without that detector appearing in the trigger pattern, the reference population being all events where at least one other detector took part in the trigger. Both Compton and coincidence techniques give consistent ionization threshold measurements within $0.2 \mathrm{keV}$. For GGA1, the values corresponding to an efficiency of $50 \%$ are $3.7 \pm 0.2$ and $3.5 \pm 0.1 \mathrm{keV}$, respectively. This represents a significant improvement compared to the performance of GeAl6, where the corresponding values varied between 5.7 and $11 \mathrm{keV}$ during the run and restricted the analysis to nuclear recoils above $30 \mathrm{keV}$. With a $50 \%$ efficiency reached at $4 \mathrm{keV}$, the nuclear recoil selection described below reaches its full efficiency within less than $1 \%$ for recoil energies above $20 \mathrm{keV}$.

The study of the distribution of the quenching factors $Q$ (the ratio of the ionization signal to the recoil energy, calculated as in [8]) recorded in the presence of a $\gamma$-ray source revealed problems with the charge collection in GeAl9 and GeAl10. Fig. 2 shows the $Q$ distribution for recoils between 20 and $200 \mathrm{keV}$ recorded with ${ }^{57,60}$ Co sources, for the different detectors. GeAl9 and GeAl10 have been added together, since they display a very similar behavior. The distributions are normalized to the number of entries in GGA1. The distribution for GGA1 is centered on 1, as expected by construction for electron recoils. The distribution for GeAl9 and GeAl10 show a narrower peak centered at 1, as expected from the better heat resolution. However, a relatively flat tail of events with $\mathrm{Q}$ values ranging from 0 to 1 is observed. While 1.3 and $2.2 \%$ of events have $Q$ values below 0.5 in GeAl9 and GeAl10, this fraction is approximately $0.01 \%$ in GGA1. The tail amplitudes do not depend on the recoil energy range. On the basis of the ${ }^{57,60} \mathrm{Co}$ calibration runs it can be expected - and later experimentally verified - that these will 
produce fake nuclear recoil events $(\mathrm{Q} \sim 0.3$, see below) at a rate of a few events per $\mathrm{kg} \cdot \mathrm{d}$ in GeAl9 and GeAl10. It was therefore decided that only the GGA1 data would be used for deriving a limit on WIMP interactions.

Although the suppression of charge collection problems in GGA1 may indicate that the amorphous layer helps prevent them, as suggested by [12], more thorough tests are needed before reaching any firm conclusion.

\section{Fiducial volume and acceptance}

As in Ref. 81, a fiducial volume is defined in order to exclude events occurring in the outer perimeter of the detector as it is more exposed to external sources of radioactivity and to charge collection problems. The selection cut is the same: more than $75 \%$ of the total charge must be collected on the center electrode. Here also, two methods are used to measure the fraction of the total detector volume thus defined. The first one uses the data collected with the ${ }^{252} \mathrm{Cf}$ source: the fraction of nuclear recoil candidates passing the fiducial cut is compared with the results of a Monte Carlo simulation of the neutron interactions in the detector. The second method exploits the uniformity of the ${ }^{65} \mathrm{Zn}$ and ${ }^{68} \mathrm{Ge}$ decays within the detector volume. The fraction of the total intensity of the 8.98 and $10.37 \mathrm{keV}$ peaks selected by the fiducial acceptance is then equal to the fiducial volume fraction. The two methods give identical results for GGA1 $(57 \pm 3 \%)$ and agree within $3 \%$ for the other two detectors.

The acceptance for nuclear recoils is defined both in terms of ranges in $Q$ and recoil energies. The neutron calibration of the three detectors confirms the parametrization used in Ref. [8], namely, the center of the band is given by $0.16\left(E_{R}\right)^{0.18}$, where $E_{R}$ is the recoil energy in $\mathrm{keV}$, and its width is equal to that predicted from the propagation of the heat and ionization resolutions added in quadrature with a constant rms spread of $\sim 0.035$. Again, the width of the band is set to $\pm 1.645 \sigma$. It was verified on the neutron data that this selection does correspond to an efficiency of $90 \%$.

The lower bound of the recoil energy range for the selection of nuclear recoil is set to $20 \mathrm{keV}$, based on the same arguments as in Ref. [8]: the efficiency to nuclear recoils should be as uniform as possible within the band, and it should exclude regions where the $\gamma$-ray rejection, estimated by propagating the experimental heat and ionization resolutions, is expected to be worse than $99.9 \%$.

Given that a background of events with improper charge collection has appeared in GeAl9 and GeAl10, and that it has a flat distribution in both $Q$ and recoil energy, one could expect a similar behavior, albeit at a lower level, in GGA1. Therefore, some care must be taken in the definition of the 
upper bound of the recoil energy range of WIMP candidates. The natural choice is to calculate, using the prescription of Ref. [9], the upper bound corresponding to $95 \%$ of all WIMP-induced recoils above $20 \mathrm{keV}$. Using the standard halo and nuclear form factor parameters the mass of the WIMP and varies from $33 \mathrm{keV}$ at $20 \mathrm{GeV} / \mathrm{c}^{2}$ to $86 \mathrm{keV}$ at 100 $\mathrm{GeV} / \mathrm{c}^{2}$ and saturates slightly above $110 \mathrm{keV}$ at masses above $10 \mathrm{TeV} / \mathrm{c}^{2}$.

Within the fiducial volume $(57 \%$ of $318.5 \mathrm{~g}$ ), the acceptance for nuclear recoils from WIMP interactions thus corresponds to $90 \%$ (width in $Q$ ) times $95 \%$ of all recoils above $20 \mathrm{keV}$ (mass-dependent recoil energy range).

\section{Results and Discussion}

The low-background physics data consists of all physics runs recorded over a period from February to May 2002. The physics data-taking period started a few months after the installation of the detectors in the Laboratoire Souterrain de Modane, after a period of optimization of resolution, calibration and of long exposure to an intense ${ }^{60} \mathrm{Co}$ source while all electrodes were shorted. The running conditions were kept as homogeneous as possible, until the run was interrupted by an accidental warm-up of the detector. In addition to a constant monitoring of the data, the homogeneity of the running conditions was checked with $\sim$ weekly ${ }^{57}$ Co calibrations and two neutron calibrations. The total physics run time at low background corresponds to 54 days, of which $2.0 \%$ are lost due to the regular shorting of the electrodes to prevent the accumulation of space charge, $3.9 \%$ are lost due to the dead-time of the data acquisition and $6.2 \%$ are lost in a few hour-long episodes where the microphonics noise reached unacceptable levels, as attested by a strong deterioration of the baseline resolutions. The total exposure is thus $15.1 \mathrm{~kg} \cdot \mathrm{d}$, of which 8.6 is in the center fiducial volume. The exposure corrected for the acceptance of the nuclear recoil band is $7.4 \mathrm{~kg} \cdot \mathrm{d}$.

The data recorded in the fiducial volume of GGA1 are shown in Fig. 3. The ionization-to-recoil energy ratios are plotted as a function of the recoil energy. Only events triggered by GGA1 alone and with an ionization energy above $3.5 \mathrm{keV}$ (hyperbolic dashed line) are shown. The $99.9 \%$ acceptance band for photons shown as a dotted line is the result of a simple propagation of the average heat and ionization resolution, assuming a Gaussian dispersion. The population of events around $\mathrm{Q} \sim 0.5$ associated with low-energy $\beta$ and $\gamma$ surface events, so prominent in our previous data of Ref. 11] and less so

1 The halo parameters are a local WIMP density of $0.3 \mathrm{GeV} / \mathrm{c}^{2} / \mathrm{cm}^{3}$, a rms velocity of $270 \mathrm{~km} / \mathrm{s}$, an escape velocity of $650 \mathrm{~km} / \mathrm{s}$ and an Earth-halo relative velocity of $230 \mathrm{~km} / \mathrm{s}$. The Helm parametrization of the nuclear form factor is used with the recommended values of $a=0.52 \mathrm{fm}, s=0.9 \mathrm{fm}$ and $c=1.23 A^{1 / 3}-0.6 \mathrm{fm}$. See Ref. [9] for details. 
in Ref. [8], is only represented here by 4 to possibly 5 events. The correct interpretation of these events would require a significant increase in exposure, given the low level of background reached with the present detector. The same is true for the interpretation of the three events below $Q=0.7$ and with recoil energies between 119 and $182 \mathrm{keV}$.

The event at $119 \mathrm{keV}$ and $Q=0.3$ is lying at $-1.646 \sigma$ of the centre of the nuclear recoil band. Given the uncertainty in the experimental determination of $\sigma$, we conservatively choose to consider this event as a nuclear recoil, entering in the acceptance for WIMP masses above $10 \mathrm{TeV} / \mathrm{c}^{2}$. For lower WIMP masses, no events are in the nuclear recoil band.

The absence of events in the defined acceptance is interpreted in terms of upper limits at 90\% CL on WIMP-nucleon scattering cross sections for $M_{W}$ $<10 \mathrm{TeV} / \mathrm{c}^{2}$ following the prescriptions of Ref. [9] with the standard halo and nuclear models described above.

The limit as a function of WIMP mass is shown in Fig. 14, where it is compared with the previous result obtained with an effective exposure of 4.3 $\mathrm{kg} \cdot \mathrm{d}$ of the GeAl6 detector [8]. The limit resulting from the combination of the two measurements, corresponding to an effective exposure of $11.7 \mathrm{~kg} \cdot \mathrm{d}$ is also shown. The $3 \sigma$ contour corresponding to the annual modulation effect of DAMA NaI1-4 [3] is shown: the black circle marks the central value of that measurement at $M_{W}=52 \mathrm{GeV} / \mathrm{c}^{2}$ and $\sigma_{n}=7.2 \times 10^{-6} \mathrm{pb}$. The present combined results are incompatible with the interpretation of the modulation effect in terms of a WIMP behaving according to the standard phenomenological model of Ref. [9]. While 9.8 nuclear recoils should have been observed between 20 and $64 \mathrm{keV}$, none are observed. The Poisson probability of such a fluctuation is $0.006 \%$. The black triangle on Fig. 因 at $M_{W}=44 \mathrm{GeV} / \mathrm{c}^{2}$ and $\sigma_{n}=5.4 \times 10^{-6} \mathrm{pb}$ corresponds to the most likely value quoted by DAMA when they combine their modulation results with their limit achieved using pulse shape discrimination in $\mathrm{NaI}$ [2]. It is also incompatible with the present EDELWEISS results, the Poisson probability of observing no events from a prediction of 6.2 events being $0.2 \%$. The only remaining part of the $3 \sigma \mathrm{NaI} 1-4$ DAMA zone corresponds to neutralino masses below the limit of $45 \mathrm{GeV} / \mathrm{c}^{2}$ obtained at LEP [14]. Clearly, the standard prescriptions of Ref. [9] fail at reconciling the EDELWEISS and DAMA experimental results.

In Fig. 5 the combined EDELWEISS limit is compared to those obtained by other direct WIMP searches. The EDELWEISS sensitivity for spinindependent WIMP-nucleon interaction is the best achieved so far by any dark matter search for masses above $35 \mathrm{GeV} / \mathrm{c}^{2}$. Furthermore, the EDELWEISS data start to probe some of the supersymmetric models predicting the highest interaction rates. Fig. 0 shows as an example the range of masses and cross-sections allowed in the calculations of Refs. [15, 16], where relaxed 
conditions of unification at the GUT scale yield higher upper bounds for $\sigma_{n}$ (for comparison, see e.g. Ref. [17]).

\section{Conclusion}

The EDELWEISS collaboration has searched for nuclear recoils due to the scattering of WIMP dark matter using a $320 \mathrm{~g}$ heat-and-ionization Ge detector operated in a low-background environment in the Laboratoire Souterrain de Modane. After a combination with our previous data [8], the achieved sensitivity is so far the best for all direct searches for WIMP masses above $35 \mathrm{GeV} / \mathrm{c}^{2}$. The limit obtained on WIMP-nucleon interaction cross-sections as a function of WIMP mass is based on the absence of events in the recoil energy range relevant for WIMP masses below $10 \mathrm{TeV} / \mathrm{c}^{2}$ and does not rely on any background subtraction. The combined EDELWEISS result is incompatible at more than $99.8 \%$ CL with a WIMP of mass $44 \mathrm{GeV} / \mathrm{c}^{2}$ and a nucleon scattering cross-section of $5.4 \times 10^{-6}$ pb reported by the experiment DAMA [3] based on the same standard nuclear physics and astrophysical assumptions. Furthermore, the EDELWEISS experiment excludes a first sample of supersymmetric models predicting the highest WIMP-nucleon interaction rates 15, 16.

\section{Acknowledgments}

The help of the staff of the Laboratoire Souterrain de Modane and of the participating laboratories is gratefully acknowledged. This work has been partially funded by the EEC Network program under contract ERBFMRXCT980167.

\section{References}

[1] L. Bergström, Rep. Prog. Phys. 63 (2000) 793;

A. Morales, VII ${ }^{\text {th }}$ Workshop on Topics in Astroparticle and Underground Physics (TAUP2001), Sept. 2001, Gran Sasso, Italy, Nucl. Phys. Proc. Suppl. 110 (2002) 39, astro-ph/0112550;

J. Ellis, IV ${ }^{\text {th }}$ Int. Heidelberg Conference on Dark Matter in Astro and Particle Physics, Feb. 2002, Cape Town, South Africa, astroph/0204059.

[2] R. Bernabei et al., Phys. Lett. B 389 (1996) 757. 
[3] R. Bernabei et al., Phys. Lett. B 480 (2000) 23.

[4] R. Abusaidi et al., Phys. Rev. Lett. 84 (2000) 5699.

[5] L. Baudis et al., Phys. Rev. D 63 (2001) 022001.

[6] A. Morales et al., Phys. Lett. B 532 (2002) 8.

[7] D. Abrams et al., Submitted to Phys. Rev. D, astro-ph/0203500.

[8] A. Benoit et al., Phys. Lett. B 513 (2001) 8, astro-ph/0106094.

[9] J.D. Lewin and P.F. Smith, Astropart. Phys. 6 (1996) 87.

[10] X.F. Navick et al., Nucl. Instr. Meth. A 444 (2000) 361.

[11] P. Di Stefano et al., Astropart. Phys. 14 (2001) 329, astro-ph/0004308;

A. Benoit et al., Phys. Lett. B 479 (2000) 8, astro-ph/0002462.

[12] P.N. Luke, C.S. Rossington and M.F. Wesela, IEEE Trans. Nucl. Sci. 41 (1994) 1074, LBNL-33980;

T. Shutt et al, Nucl. Instr. Meth. A 444 (2000) 340.

[13] M.C. Lederer and V.S. Shirley, Table of isotopes (VII ${ }^{\text {th }}$ edition), John Wiley \& Sons (New York) 1978.

[14] I. Laktineh, Proceedings of the XXXVII Rencontres de Moriond, Les Arcs, France (2002), hep-ex/0205088.

[15] A. Bottino, F. Donato, N. Fornengo and S. Scopel, Phys. Rev. D 63 (2001) 125003.

[16] E.A. Baltz and P. Gondolo, Phys. Rev. Lett. 86 (2001) 5004.

[17] J. Ellis, A. Ferstl and K.A. Olive, Phys. Lett. B 481 (2001) 304. 


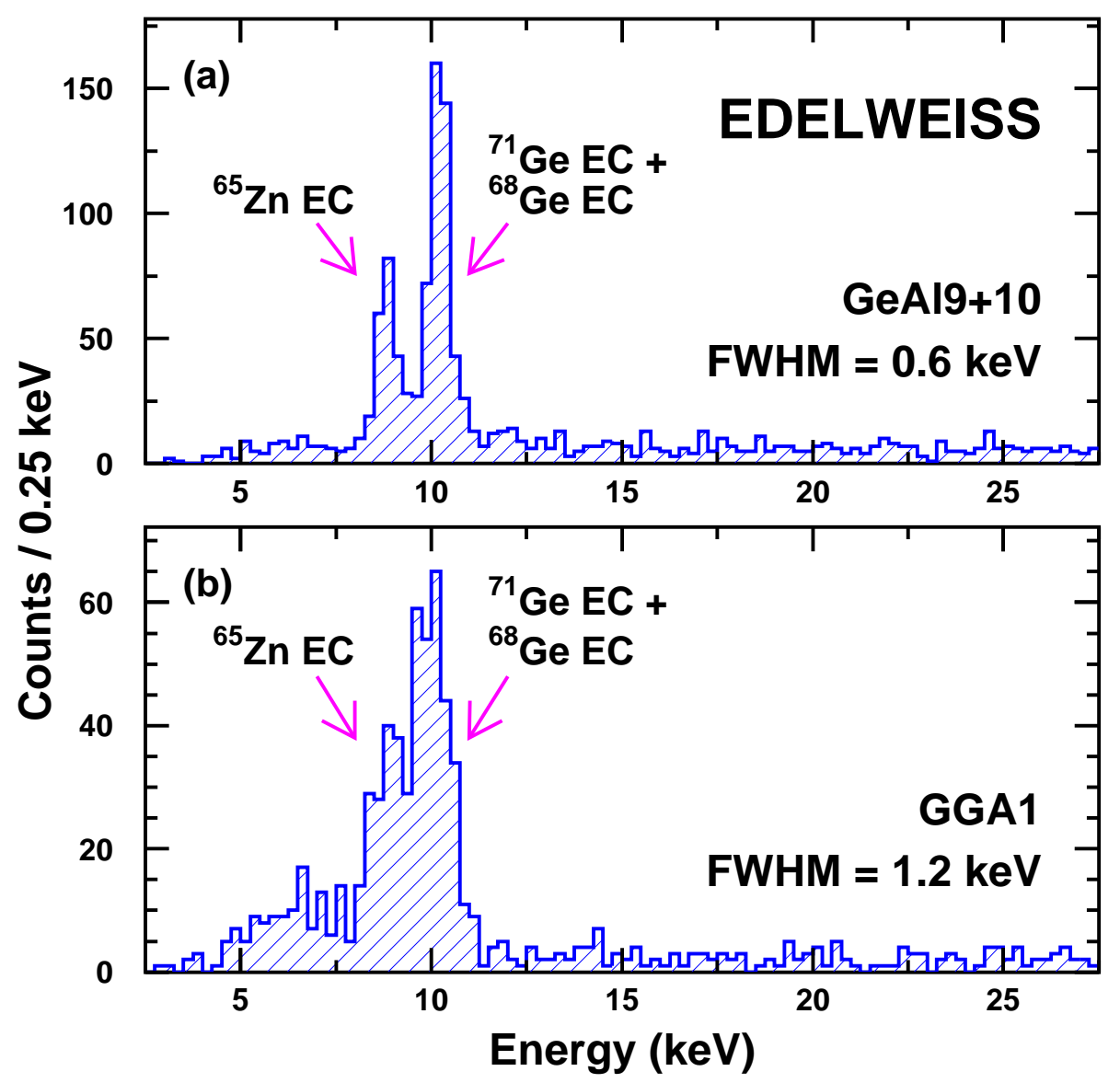

Figure 1: Energy pulse height spectra for low-energy gammas (sum of the ionization and heat channels, weighted by their resolution squared) in the fiducial volume of the EDELWEISS detector, for the low-background physics runs: (a) sum of the distributions of the detectors GeAl9 and GeAl10; (b) distribution in the detector GGA1. The arrows indicate the peaks at 8.98 and $10.37 \mathrm{keV}$, corresponding to the de-excitation of the cosmogenic activation of ${ }^{65} \mathrm{Zn}$ and ${ }^{68} \mathrm{Ge}$ in the detector, and the ${ }^{71} \mathrm{Ge}$ activation that follows neutron calibrations. 


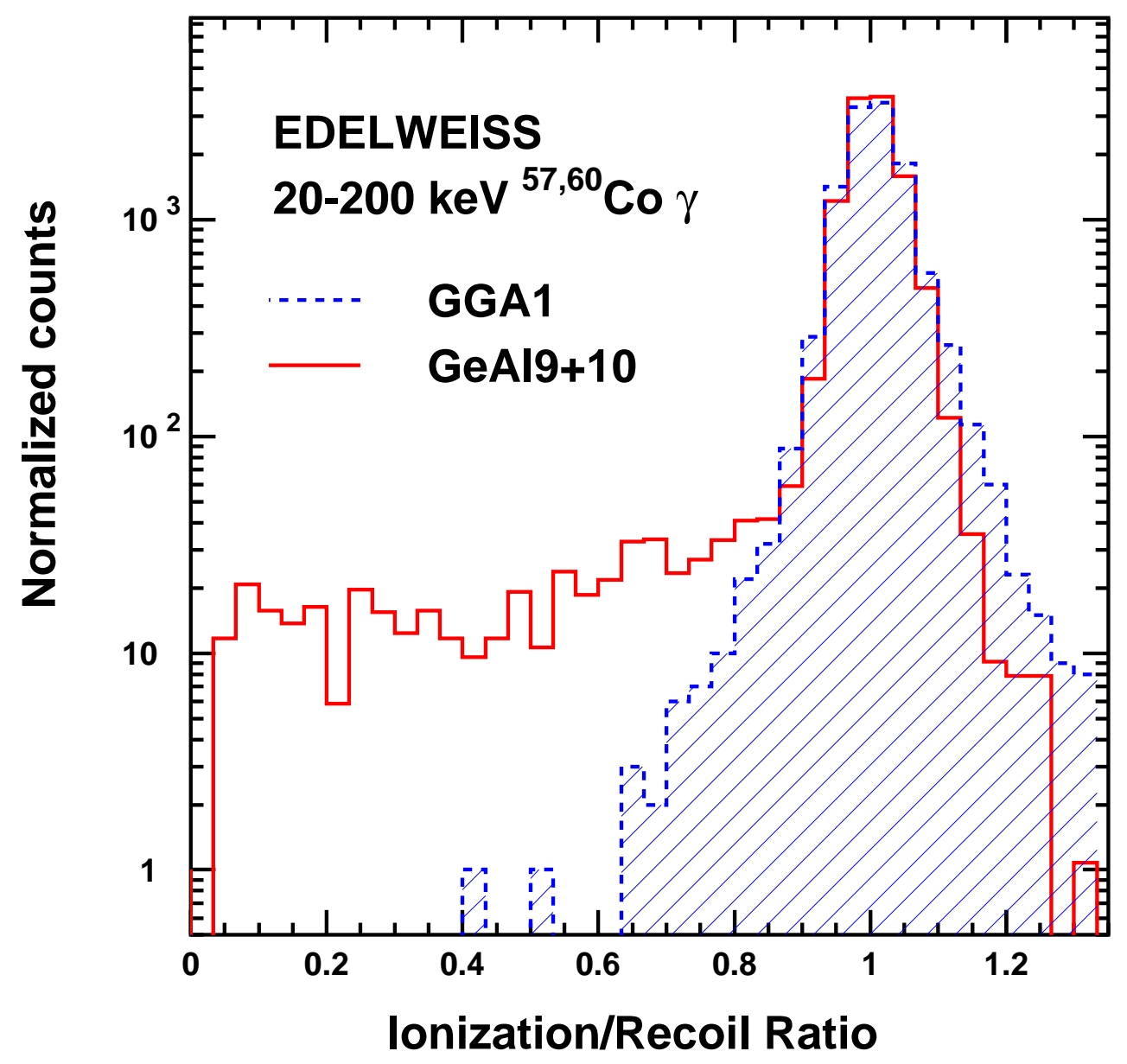

Figure 2: Distribution of the ratio of the ionization pulse height to the recoil energy (quenching factor Q) obtained by exposing the detectors to ${ }^{57} \mathrm{Co}$ and ${ }^{60} \mathrm{Co} \gamma$-ray sources. The ratio is normalized to 1 for electron recoils using the photopeaks of the ${ }^{57}$ Co source. Shaded histogram: detector GGA1. Line histogram: sum of the detector GeAl9 and GeAl10, normalized to the same number of entries of GGA1. 


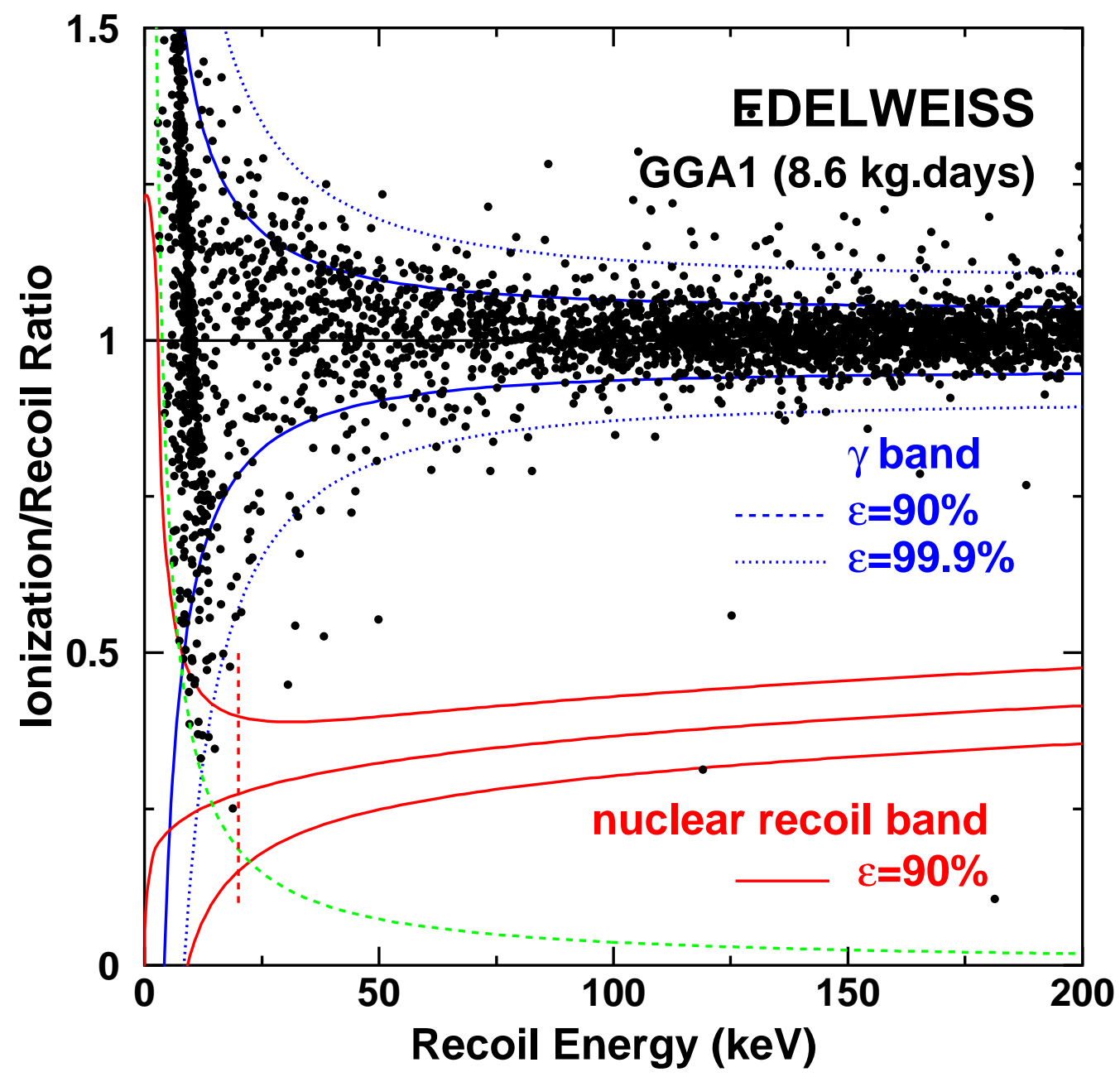

Figure 3: Distribution of the quenching factor (ratio of the ionization signal to the recoil energy) as a function of the recoil energy from the data collected in the center fiducial volume of the $320 \mathrm{~g}$ EDELWEISS detector GGA1. The exposure of the fiducial volume corresponds to $8.6 \mathrm{~kg} \cdot \mathrm{d}$. Also plotted as full lines are the $\pm 1.645 \sigma$ bands ( $90 \%$ efficiency) for photons and for nuclear recoils. The $99.9 \%$ efficiency region for photons is also shown (dotted line). The hyperbolic dashed curve corresponds to $3.5 \mathrm{keV}$ ionization energy and the vertical dashed line to $20 \mathrm{keV}$ recoil energy. 


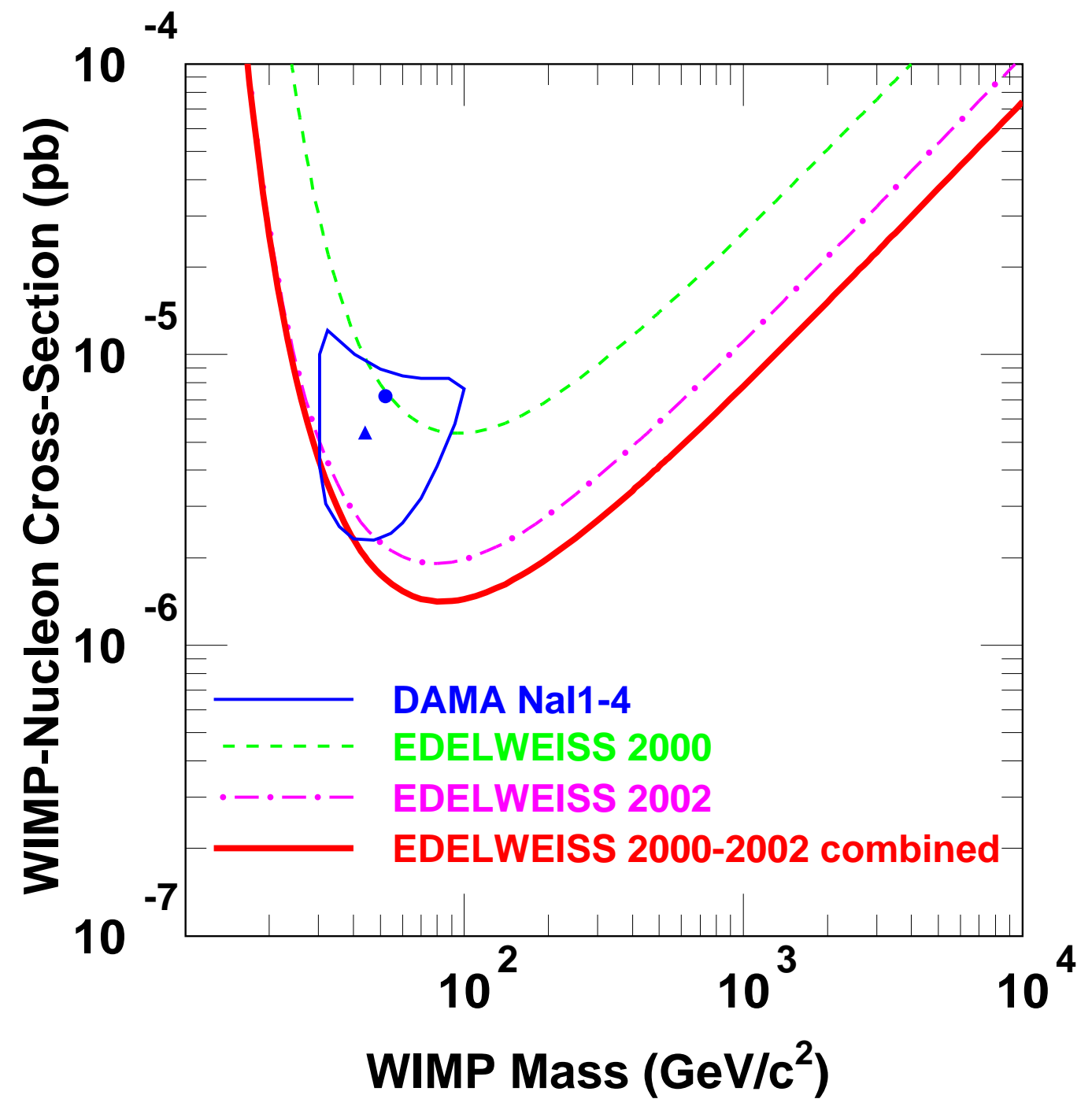

Figure 4: Spin-independent exclusion limits (dark solid curve) obtained by combining our 2000 data from Ref. [8] with the present data, for a total exposure of $11.7 \mathrm{~kg} \cdot \mathrm{d}$. Dashed curve: previous EDELWEISS data [8] reanalyzed using the new definition of the upper bound of the recoil energy range (acceptance of 95\%). Dash-dotted curve: present 2002 data. Closed contour: allowed region at $3 \sigma$ CL from the DAMA1-4 annual modulation data [3]. The full circle and triangle within this contour are defined in the text. 


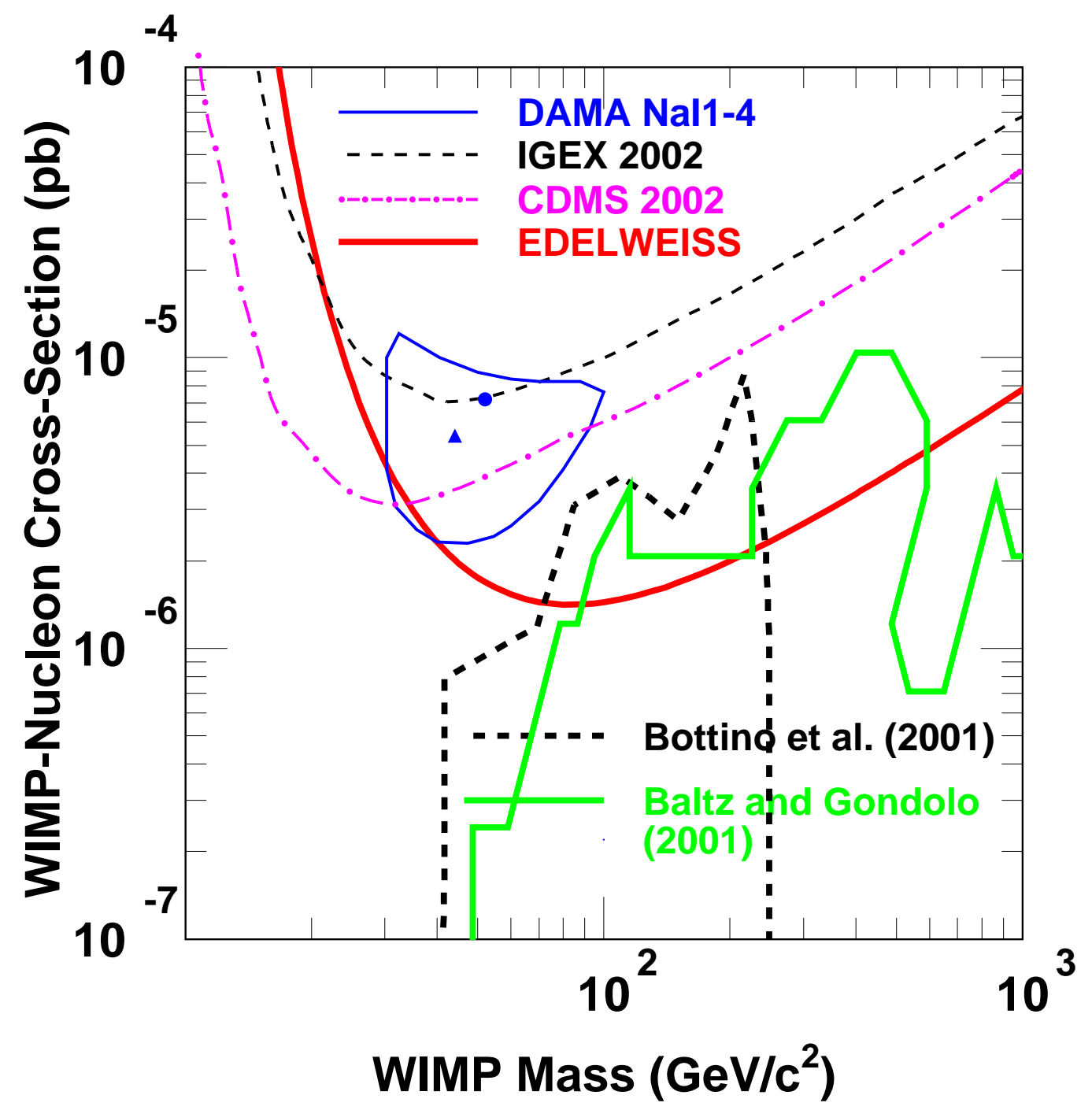

Figure 5: Combined EDELWEISS spin-independent exclusion limits (dark solid curve) compared with published limits from other experiments and theoretical calculations. Dashed curve: Ge diode limit from IGEX [6]. Dashdotted curve: CDMS limit with statistical subtraction of the neutron background [7]. Closed contour: allowed region at $3 \sigma$ CL from the DAMA1-4 annual modulation data [3]. Two regions spanned by some of the supersymmetric model calculations of Refs. [15, 16] are also shown. 